\title{
Titanium Plate Cranioplasty Induced Intravascular Papillary Endothelial Tumor: Case Report and Review of the Literature
}

\author{
Uri P Hadelsberg*, Nevo Margalit and Lior Gonen \\ Department of Neurosurgery, Israel
}

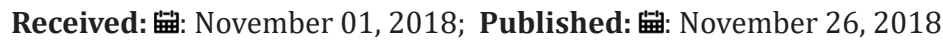

*Corresponding author: Uri P Hadelsberg, Department of Neurosurgery, Jerusalem, Israel

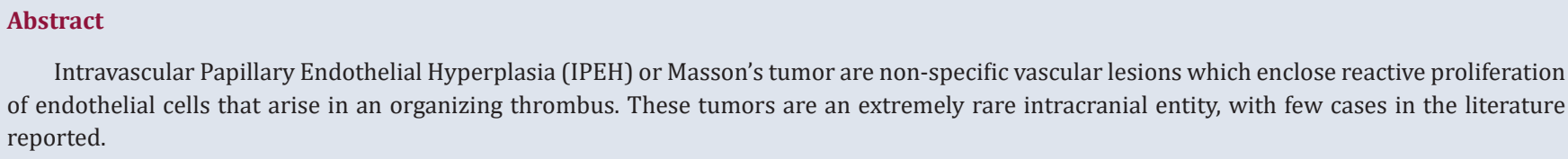

Intravascular Papillary Endothelial Hyperplasia (IPEH) or Masson's tumor are non-specific vascular lesions which enclose reactive proliferation of endothelial cells that arise in an organizing thrombus. These tumors are an extremely rare intracranial entity, with few cases in the literature reported.

Case Report: The authors present a 66-year-old male who underwent decompressive craniectomy and a titanium plate cranioplasty due to a warfare injury 40 years ago. Recently, he presented to our institution with a lesion which has started to grow several years prior to his admission, growing on the patient's titanium plate underneath the skin. He underwent surgery with complete removal of the tumor and made a complete recovery. The tumor turned out to be an Intravascular Papillary Endothelial Hyperplasia (IPEH) tumor. The authors present a case report involving an intracranial IPEH tumor and current intracranial treatment modalities for this type of tumor.

Abbreviations: IPEH: Intravascular Papillary Endothelial Hyperplasia; CT: Computed Tomography; IPEHT: IPEH Tumors; MRI: Magnetic Resonance Imaging

\section{Case Report}

The authors present a craniotomy for removal of a left frontal lesion growing on a titanium cranioplasty. This 66-year-old doctor was injured in the Ararb-Israeli 1973 War. He was a tank driver and his tank were the last tank to suffer a direct hit from a mortar bomb shell during the war. He underwent urgent surgery and a right frontal craniectomy in Egypt, leaving a titanium plate as a bone graft instead of his frontal bone which was removed during surgery. 4 years prior to this admission, a frontal subcutaneous bossing on the patent's frontal bone appeared. It was indolent until 2 years prior to admission when it demonstrated continued growth. The tumor's last dimensions prior to surgery were measured at $9 \mathrm{~cm}$ width over $5 \mathrm{~cm}$ length. This 66-year-old male sustained a head injury in 1973. However, he continued to live a normal, fulfilling life. Being the first student in his class to graduate medical school after being in a rehab institution for combat soldiers. The patient was recently admitted to our service to undergo surgery for removal of a superficial subcutaneous lesion which presented as a cosmetic problem. The patient denied headaches, seizures or any other neurological complaint, however the appearance greatly disturbed him, enough to undergo surgery and removal of the lesion. Prior to surgery, the patient underwent a Computed Tomography (CT) scan which demonstrated an extra-axial lesion adherent to the patient's titanium plate (Figures $1 \& 2$ ).

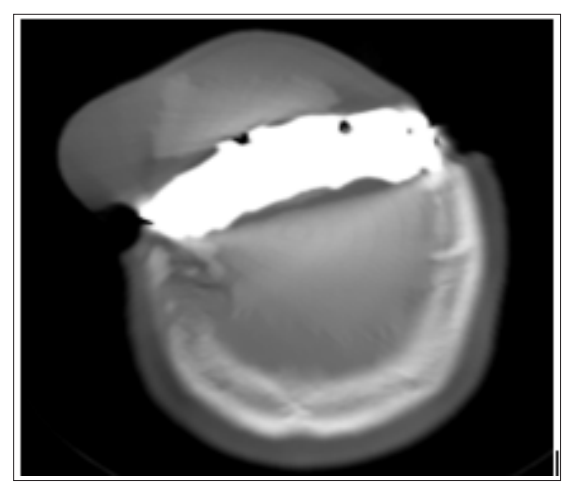

Figure 1: An axial CT scan in the bone window displaying the patient's lesion which can be seen on top of the patient's titanium plate. 


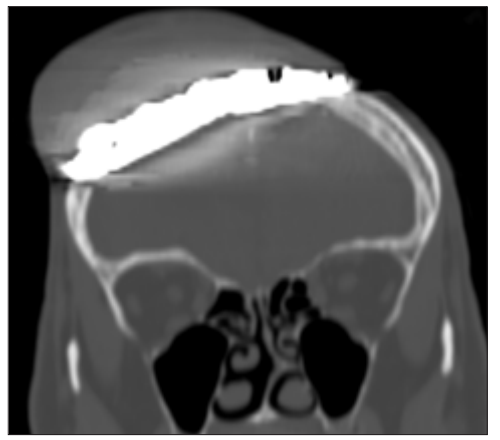

Figure 2: A coronal CT scan displaying the same lesion.

During surgery, the patient was laid in the supine position (Figures 3 \& 4) and an incision on the skin immediately superior to where the tumor was palpated was made. The tumor was quickly visualized with a gross total excision of the tumor via an approachable and well-defined plain achieved (Figures 5 \& 6). The titanium plate inserted during his first operation was made visible during surgery (Figure 7). Suturing of the scar with complete closure was done and a temporary drain left subcutaneously (Figure 8). The patient was extubated in the operating room with no immediate neurological deficit. After surgery, the patient underwent a swift recovery. He was discharged after several days with no neurological deficits, and in an elated spirit after removal of this subcutaneous lesion. The pathology report turned out to be of an Intravascular Papillary Endothelial Hyperplasia (IPEH) type defined as a Masson tumor.

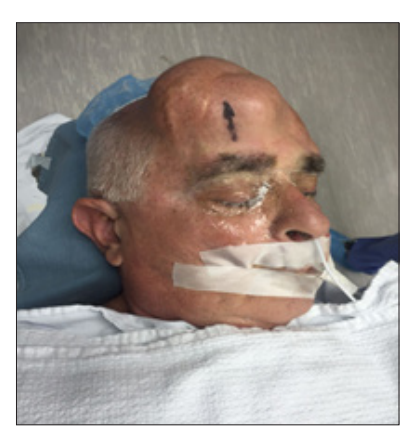

Figure 3: Anterior view of patient's face and tumor in the operating room.

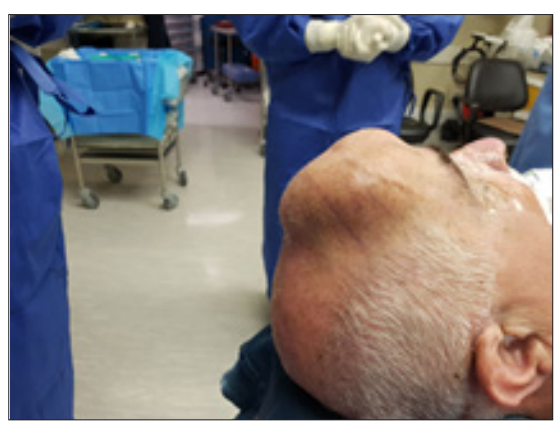

Figure 4: Lateral view of patient's face and tumor in the operating room.

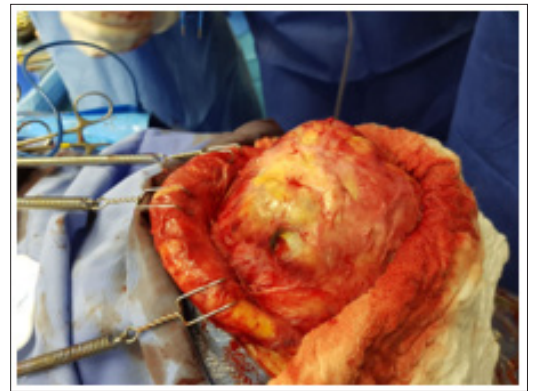

Figure 5: View of the tumor during surgery.

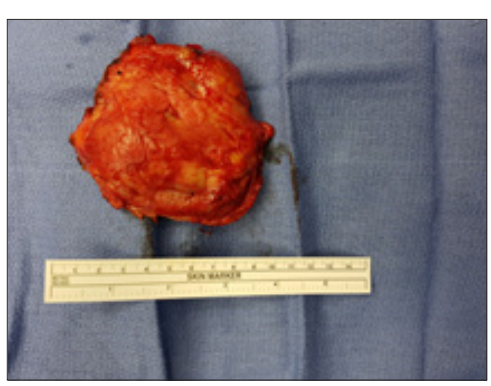

Figure 6: View of excised tumor.

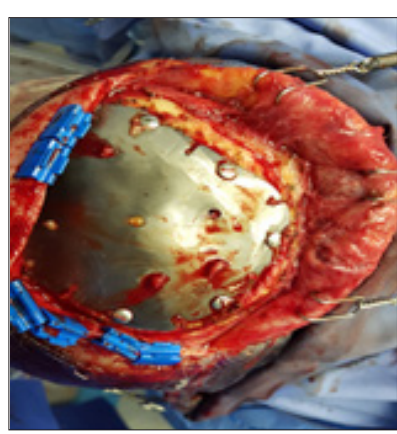

Figure 7: View of titanium plate without the tumor.

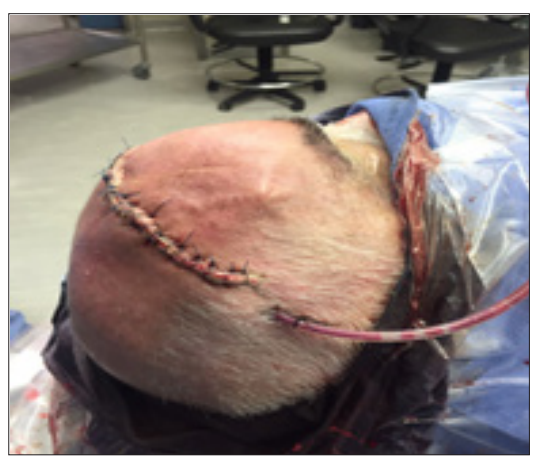

Figure 8: Lateral view of patient's face after tumor excision and skin closure.

\section{Discussion}

IPEH tumors were first described by Masson in 192310-12. Papillary endothelial hyperplasia tumors are a benign, non-specific vascular lesion which enclose reactive proliferation of endothelial 
cells that arise in an organizing thrombus [1]. The term IPEH suggests that thrombosis happens prior to papillary proliferation and that this proliferation is aided by the thrombotic material's matrix [2]. IPEH Tumors (IPEHT) are most commonly seen in the skin's sub-cutaneous tissue with intracranial lesions being rare, with a bit over 30 cases which have hitherto been reported. On Computed Tomography (CT) imaging, these tumors pose a diagnostic challenge, with difficulty telling apart these tumors and eosinophilic granulomas or other skull tumors. In addition, on CT scans hemorrhage is commonly present [3], however no hemorrhagic signs were seen on this patient's CT scan. In addition, Magnetic Resonance Imaging (MRI) scans can demonstrate the extremely vascular and hyperdense nature of IPEHTs93. Imaging can be misleading, however, as Ong et al. report on a 37-yearold male which underwent surgery for resection of a WHO grade III anaplastic astrocytoma 10. 5 years into follow-up, the patient's imaging showed progressive enlargement of the lesion into the resection cavity, radiographically consistent with tumor recurrence. Chemotherapy was considered, but eventually the patient underwent surgical excision with gross total resection and the pathology was consistent with an IPEHT.

Cagli and colleagues reported about 4 intracranial cases of PEHs which were treated surgically. They report complete removal of only 2 of the lesions, however all 4 cases involved cranial nerve deficits [4]. Furthermore, surgical removal, when complete seems to be enough treatment $[3,5,6]$. It is important to completely remove the tumor as recurrence or progression may be seen. As an example, for difficult to treat tumors of this type, Avellino et al. report of a Masson tumor in the cerebellopontine angle of a 75-year-old woman. The patient underwent preoperative embolization, subtotal resection and postoperative radiotherapy. The patient was symptomatic 9 years later and had to undergo a repeat embolization and near gross total resection. 15 months after her first recurrence, a second recurrence was seen to which she underwent stereotactic gamma knife surgery, leaving a residual and stable looking tumor on follow up of 2.75 years. Indeed, Hastruk et al. also reported on an IPEH tumor mimicking a recurrence in a 56-year-old male which was operated for a left posterior convexity meningioma and which was thought to have a recurrence [7]. In cases where the surgical approach is challenging, unlike in our case, Gamma Knife radiosurgery can be employed. Ohshima et al. report of a successful treatment of a superior orbital fissure PEHT after incomplete resection of the tumor as an alternative therapeutic modality [8], however due to a small cohort and few cases reported of radiosurgery treatment, it is difficult to draw conclusions about the efficacy of adjuvant treatment [3].

The use of titanium cranioplasties for calvarial defects is still seen today [9], with long term outcomes of patients undergoing secondary cranioplasty after decompressive craniectomy for trauma or infraction being the material of choice. However, there are no reports of lesions growing on top of titanium cranioplasties. In a prospective trial from July 2018, comparing long term outcomes of cranioplasty with the use of an autologous cranioplasty and custom-made titanium bones in patients after craniectomies, custom-made titanium skulls had fewer bone resorption [10]. However, complications are seen with the use of this material. Hill et al. [11] retrospectively examined all titanium cranioplasties in his institution between 2007-2011, with 95 patients included in the study. 29 of these patients developed one or more post-cranioplasty complications, with a complication rate of $31.5 \%$. The following complications were noted: subdural hematoma, extradural hematoma, CSF leak, seizures, intracerebral hemorrhage [12], subgaleal collection and pain. Of these 29 cases, 9 required cranioplasty removal and 10 required a further surgical procedure, with infection being the most common complication. In another retrospective study of titanium cranioplasties involving 174 patients, Mukherjee et al. had a complication rate of $26.4 \%$ and $10.3 \%$ rate of plate removal $[13,14]$. In neither studies were there any abnormal lesions growing on the plate.

\section{Conclusion}

To the best of our knowledge, this is the first case of an IPEH tumor occurring after secondary cranioplasty due to combatinduced craniectomy. IPEH tumors are a rare intracranial entity, which may also cause dire cosmetic consequences. These tumors, however, when located on the superficial brain, can be successfully fully removed. In cases of incomplete intracranial removal or where cranial nerves are involved, one may use additional treatment modalities such as radiosurgery after a surgical attempt. Further research with larger cohorts of patients are needed in-order to understand the role of adjuvant therapy in these rare intracranial tumors.

\section{References}

1. Tosios K, Koutlas IG (1994) Papanicolaou SI: Intravascular papillary endothelial hyperplasia of the oral soft tissues: Report of 18 cases and review of the literature. J Oral Maxillofac Surg 52(12): 1263-1268.

2. Clearkin KP, Enzinger F (1976) Intravascular papillary endothelial hyperplasia. Arch Pathol Lab Med 100(8): 441-444.

3. Shah HC, Mittal DH, Shah JK (2014) Intravascular papillary endothelial hyperplasia (Masson's tumor) of the scalp with intracranial extension. J Pediatr Neurosci 9(3): 260-262.

4. Cagli S, Oktar N, Dalbasti T, Işlekel S, Demirtaş E (2004) Intravascular papillary endothelial hyperplasia of the central nervous system four case reports. Neurol Med Chir (Tokyo) 44(6): 302-310.

5. Ong SS, Bruner J, Schellingerhout D, Puduvalli VK (2011)Papillary endothelial hyperplasia presenting as recurrent malignant glioma. J Neurooncol 102(3): 491-498.

6. Park KK, Won YS, Yang JY, Choi CS, Han KY (2012) Intravascular papillary endothelial hyperplasia (Masson tumor) of the skull: Case report and literature review. J Korean Neurosurg Soc 52(1): 52-54.

7. Hasturk AE, Basmaci M (2013) Foreign body granuloma mimicking recurrent intracranial tumor: A very rare clinical entity. Acta Med Iran 51(11): 816-818.

8. Ohshima T, Ogura K, Nakayashiki N, Tachibana E (2005) Intravascular papillary endothelial hyperplasia at the superior orbital fissure: report of a case successfully treated with gamma knife radiosurgery. Surg Neurol 64(3): 266-269.

9. Williams L, Fan K, Bentley R (2016) Titanium cranioplasty in children and adolescents. J Cranio-Maxillofacial Surg 44(7): 789-794. 
10. Honeybul S, Morrison DA, Ho KM, Lind CRP, Geelhoed E (2018) A randomised controlled trial comparing autologous cranioplasty with custom-made titanium cranioplasty: Long-term follow-up. Acta Neurochir (Wien) 160(5): 885-891.

11. Hill CS, Luoma AMV, Wilson SR, Kitchen N (2012) Titanium cranioplasty and the prediction of complications. Br J Neurosurg 26(6): 832-837.

12. Avellino AM, Grant GA, Harris AB, Wallace SK, Shaw CM (1999) Recurrent intracranial Masson's vegetant intravascular hemangioendothelioma. J Neurosurg 91(2): 308-312.

\section{ISSN: 2574-1241}

DOI: 10.26717/BJSTR.2018.11.002089

Uri P Hadelsberg. Biomed J Sci \& Tech Res

(C) This work is licensed under Creative

Submission Link: https://biomedres.us/submit-manuscript.php
13. Cabraja M, Klein M, Lehmann TN (2009) Long-term results following titanium cranioplasty of large skull defects. Neurosurg Focus 26(6): E10.

14. Mukherjee S, Thakur B, Haq I, Hettige S, Martin AJ (2014) Complications of titanium cranioplasty-a retrospective analysis of 174 patients. Acta Neurochir (Wien) 156(5): 989-998.

$\begin{array}{ll}\text { BIOMEDICAL } & \text { Assets of Publishing with us } \\ \text { RESEARCHES } & \text { - Global archiving of articles } \\ \text { - Immediate, unrestricted online access }\end{array}$

